\title{
Another Consequence of Obesity: Chronic Traumatic Encephalopathy
}

\author{
Scott A. Shikora ${ }^{1,2}$ \\ Published online: 20 October 2020 \\ (C) Springer Science+Business Media, LLC, part of Springer Nature 2020
}

\section{EDITORIAL}

Go Ahead and Add Chronic Traumatic Encephalopathy to the list of the Consequences of Obesity...

By the time you read this, we will have neared the end of 2020 - a year that has seen more than its share of changes. Weight loss surgery has matured into bariatric surgery, and now, we proclaim it as metabolic surgery. Along with the evolving name, the discipline has dramatically increased in size, as the safety and efficacy of the bariatric/metabolic surgical (BMS) procedures have become increasingly refined. With that in mind, are there any clinicians worldwide that do not understand the association between obesity and a wide range of comorbid conditions including type 2 diabetes, hypertension, etc.? In fact, when I speak to trainees and medical colleagues, I often use the line, "All organ systems of the body are adversely affected by obesity." For intracranial consequences of obesity, my talks and my slides discuss "Idiopathic Intracranial Hypertension (Pseudotumor Cerebri)." Furthermore, I explain that there is a wealth of published outcome studies to convince even the most doubting individual that BMS can improve or even "cure" most of these conditions.

So why am I preaching to the choir?

Do you routinely mention chronic traumatic encephalopathy when you discuss the obesity-related comorbid conditions? Furthermore, do you mention, that like the other aforementioned conditions, weight loss and BMS can be beneficial for patients suffering from obesity and chronic traumatic brain injury? I certainly did not know that.

I am excited to announce that we are publishing an article written by Professor Henry Buchwald and colleagues that will

Scott A. Shikora

sshikora@BWH.Harvard.edu

1 Harvard Medical School, Boston, MA, USA

2 Center for Metabolic and Bariatric Surgery, Brigham and Women's Hospital, 75 Francis Street, Boston, MA 02115, USA likely be the most thorough review article on this topic written to date [1]. The over 300 references add substance and validity to the subject materials. The article thoroughly covers this subject describing the risk of developing obesity after suffering a traumatic brain injury and the adverse effects of obesity on chronic brain-injured patients. It concludes with a review of the benefits of weight loss and BMS and presents data that BMS can result in the improvement of brain function and possibly even the reversal of brain lesions.

This paper is a must-read for all clinicians involved with the care of patients suffering from obesity, and those clinicians who care for brain-injured patients. However, the manuscript is lengthy, so we have elected to divide the paper into three parts and, starting with this issue, publish one part per monthly journal issue for three issues. I am confident that you will find this paper to be as interesting as I did. After you read it, let me know what you think.

Warm regards and wishes for all of you to have a better year in 2021 than that which we just experienced.

Scott A. Shikora, MD, FACS, FASMB.

\section{Reference}

1. McGlennon TW, Buchwald JN, Pories WJ, Yu F, Roberts A, Ahnfeldt EP, Buchwald H. Bypassing TBI: metabolic surgery and the link between obesity and traumatic brain injury - a review (part 1), 2020: XX,XX, Obes Surg

Publisher's Note Springer Nature remains neutral with regard to jurisdictional claims in published maps and institutional affiliations. 